\begin{tabular}{|c|c|c|}
\hline \multirow{3}{*}{$\begin{array}{r}\text { Case Reports in } \\
\text { Gastroenterology }\end{array}$} & \multirow{2}{*}{\multicolumn{2}{|c|}{ Case Rep Gastroenterol 2016;10:431-439 }} \\
\hline & & \\
\hline & $\begin{array}{l}\text { DOI: } 10.1159 / 000446641 \\
\text { Publisnea onine: August 12, } 2016\end{array}$ & $\begin{array}{l}\text { ( ) } 2016 \text { The Author(s) } \\
\text { Published by S. Karger AG, Basel } \\
\text { www.karger.com/crg }\end{array}$ \\
\hline & \multicolumn{2}{|c|}{$\begin{array}{l}\text { This article is licensed under the Creative Commons Attribution-NonCommercial } 4.0 \\
\text { International License (CC BY-NC) (http://www.karger.com/Services/OpenAccessLicense) } \\
\text { Usage and distribution for commercial purposes requires written permission. }\end{array}$} \\
\hline
\end{tabular}

\title{
Presence of Cytokeratin 19-Expressing Cholangiocarcinoma-Like Tumour in a Liver Metastatic Lesion of Rectal Neuroendocrine Tumour
}

\author{
Norihiro Ishii ${ }^{a, b} \quad$ Kenichiro Araki ${ }^{a, b} \quad$ Takehiko Yokobori ${ }^{c}$ \\ Mariko Tsukagoshi ${ }^{a, b}$ Takamichi Igarashi ${ }^{b}$ Akira Watanabe $^{a, b}$ \\ Norio Kubo ${ }^{a, b}$ Keitaro Hirai $^{b}$ Ken Shirabe $^{b}$ Hiroyuki Kuwano $^{a}$ \\ ${ }^{a}$ Department of General Surgical Science, Gunma University, Graduate School of \\ Medicine, Maebashi, Japan; ${ }^{b}$ Department of Hepatobiliary and Pancreatic Surgery, \\ Gunma University, Graduate School of Medicine, Maebashi, Japan; 'Department of \\ Molecular Pharmacology and Oncology, Gunma University, Graduate School of Medicine, \\ Maebashi, Japan
}

\section{Keywords}

Mixed adenoneuroendocrine carcinoma $\cdot$ Adenocarcinoma $\cdot$ Bile duct $\cdot$ Stem cell .

Carcinogenesis · Amphicrine tumour

\begin{abstract}
Introduction: Tumours with adenocarcinoma and neuroendocrine components have often been reported, although the reason underlying the dual components remains unclear. Case Presentation: A 43-year-old woman with multiple liver metastatic lesions of rectal neuroendocrine tumour underwent primary tumour resection and subsequent liver transplantation. Pathological examination indicated a cholangiocarcinoma-like tumour with gland formation, adjacent to a liver metastatic lesion of the neuroendocrine tumour. This tumour comprised atypical columnar epithelium, and stained positively for neuroendocrine markers and the ductal marker cytokeratin 19, indicating amphicrine properties and a partial cholangiocarcinoma phenotype - features not observed in the primary and metastatic neuroendocrine tumours. Conclusion: The presence of adenocarcinoma only at the metastatic site indicated
\end{abstract}




\section{Case Reports in Gastroenterology}

Case Rep Gastroenterol 2016;10:431-439 $10.1159 / 000446641$ www.karger.com/crg

Ishii et al:: Presence of Cytokeratin 19-Expressing Cholangiocarcinoma-Like Tumour in a Liver Metastatic Lesion of Rectal Neuroendocrine Tumour

that neuroendocrine tumour cells acquired stemness and differentiated into adenocarcinoma through metastasis, or that the adenocarcinoma newly arose from the adjacent epithelium influenced by the neuroendocrine tumour. We propose a novel mechanism for the pathogenesis of mixed tumours in neuroendocrine tumours.

\section{Introduction}

Although cases of gastroenteropancreatic neuroendocrine tumours are relatively rare, the prevalence of this condition is increasing worldwide [1]. Neuroendocrine tumours are believed to be derived from neuroendocrine cells present in mucosa and submucosa. According to the latest WHO classification, neuroendocrine tumours are classified as G1, G2, and neuroendocrine carcinoma based on the histopathological evaluation of the mitotic and Ki-67 labelling index. Moreover, the tumours with dual adenocarcinomatous and neuroendocrine differentiation, wherein each component represents at least $30 \%$ of the tumour, are classified as mixed adenoneuroendocrine carcinoma (MANEC) [2]. Cases of MANEC are very rare. Some reports have described the coexistence of adenocarcinoma and neuroendocrine components in the same tumour, even in cases where the tumours did not meet the MANEC definition [3, 4]. In previous reports, it was suggested that the adenocarcinoma component transforms or differentiates into the neuroendocrine phenotype through the analysis of DNA mutation status in each component $[3,5]$, or that tumours with both adenocarcinoma and neuroendocrine components arise from multipotent stem cells [6]. Nevertheless, the reasons underlying the coexistence of the adenocarcinoma and neuroendocrine components within the same tumour remain unclear.

In the present report, we hypothesise a novel mechanism wherein the adenocarcinoma component arises from a pure neuroendocrine tumour or from epithelial cells adjacent to the neuroendocrine tumour. To confirm our above-mentioned hypothesis, we performed a detailed pathological examination of the metastatic tumours from a primary neuroendocrine tumour without any adenocarcinoma component, to exclude the possibility of transformation or differentiation from adenocarcinoma. Here, we describe a case of dual small adenocarcinoma and neuroendocrine components in a liver metastatic lesion from a rectal neuroendocrine tumour. The dual-component feature in the liver metastatic lesion was in fact not observed in the primary tumour, indicating that the adenocarcinoma component may have arisen from the neuroendocrine tumour or neighbouring epithelial cells.

\section{Case Summary}

A 43-year-old woman was found to have swelling of the liver during an annual health check. She had a history of bronchial asthma since childhood. The laboratory examination findings were almost normal, and the levels of tumour markers, including carcinoembryonic antigen and carbohydrate antigen 19-9, were normal as well. However, the levels of neuronspecific enolase were elevated $(46.1 \mathrm{ng} / \mathrm{ml})$. Ultrasonography and computed tomography indicated multiple nodules in the liver, which suggested the presence of multiple liver metastases. Therefore, we performed whole body screening. Colonoscopy showed an elevated tumour measuring approximately $3.0 \mathrm{~cm}$ in the lower rectum, and hence, a biopsy was performed. Examination of the biopsy specimen indicated a diagnosis of neuroendocrine tumour. Moreover, the biopsy specimen of the liver tumour exhibited findings consistent with 
a neuroendocrine tumour of the rectum. However, no metastasis to other distant organs was detected. Therefore, surgery was scheduled, both in the rectum and liver in a 2-stage operation, followed by liver transplantation. Accordingly, the patient initially underwent low anterior resection, and liver transplantation was then performed from a living donor (her son) at 2 months after the first operation.

\section{Pathological Findings}

A paraffin-embedded block of the specimen was cut into 2- $\mu$ m-thick sections, which were mounted on glass slides for haematoxylin and eosin (HE) staining and immunohistochemical (IHC) staining.

The primary rectal tumour that spread to the submucosa measured $5.5 \times 5.5 \mathrm{~cm}$. HE staining indicated that the tumour comprised small nests or palisading patterns, wherein the tumour cells were relatively small and uniform, with round nuclei and prominent nucleoli (fig. 1a, b). IHC staining showed that the tumour cells were positive for synaptophysin and chromogranin A (fig. 1c, d). The Ki-67 labelling index was $<1 \%$ (fig. 1e). Although strong invasion towards the serosa, perivascular/perineural invasion, and lymph node metastases were observed, the surgical margin was negative. Moreover, no evidence of an adenocarcinoma component was identified. Based on these pathological findings, the tumour was diagnosed as carcinoid of the rectum at that time; at present, according to the WHO classification, it can be classified as neuroendocrine tumour, G1.

Thereafter, a detailed pathological examination of the liver metastatic lesions of the neuroendocrine tumour and the tissue surrounding the tumours was performed. Numerous liver metastatic lesions were noted in this case (fig. 2a). Among these tumours, a total of 14 metastatic lesions were evaluated by using HE and IHC staining. In one of these tumours, we detected a microscopic cholangiocarcinoma-like tumour that had histological features different from the adjacent metastatic lesion (fig. 2b). The tumour of the liver metastatic lesion was composed of small cuboidal cells with round nuclei and basophilic cytoplasm that formed pseudo-rosettes or a palisading pattern, consistent with primary neuroendocrine tumour (fig. 2c). However, the cholangiocarcinoma-like tumour consisted of columnarshaped cells with a slightly irregular nucleus, wherein the nuclear cytoplasmic ratio was small and the polarity was relatively maintained. The cells of the cholangiocarcinoma-like tumour were larger than those of the metastatic lesion. Moreover, focal gland formation within the cholangiocarcinoma-like tumour was observed, which resembled a welldifferentiated ductal carcinoma (fig. 2d). On IHC staining, the metastatic lesion and cholangiocarcinoma-like tumour were found to be positive for synaptophysin, chromogranin $\mathrm{A}$, and p53 (fig. 3a, b). Although the results for neuroendocrine markers via IHC staining were similar for the tumours, the expression of ductal marker cytokeratin 19 (CK19) was markedly different. The primary tumour and liver metastatic lesions were negative for CK19 (fig. 4a, b), whereas the cholangiocarcinoma-like tumour was diffusely positive for CK19 (fig. 4c). Thus, we believe that the cholangiocarcinoma-like tumour comprised an adenocarcinoma component.

Furthermore, a normal bile duct was observed within the cholangiocarcinoma-like tumour (fig. 4c, arrows), and interestingly, a region that appeared to represent a transition from bile duct to cholangiocarcinoma-like tumour was also observed (fig. 4d). This finding was not observed in the liver metastatic lesions of the neuroendocrine tumour. 


\section{Case Reports in \\ Gastroenterology}

Case Rep Gastroenterol 2016;10:431-439 $10.1159 / 000446641$

(C) 2016 The Author(s). Published by S. Karger AG, Basel www.karger.com/crg

Ishii et al.: Presence of Cytokeratin 19-Expressing Cholangiocarcinoma-Like Tumour in a Liver Metastatic Lesion of Rectal Neuroendocrine Tumour

\section{Discussion}

According to the latest WHO classification, tumours exhibiting both adenocarcinoma and neuroendocrine components are termed MANEC [2]. Based on the guidelines for MANEC classification, each component must comprise at least $30 \%$ of the tumour; however, tumours with adenocarcinoma and neuroendocrine components in various proportions have often been reported [4]. In 1987, Lewin [7] first proposed the classification of such tumours into 3 different subtypes: mixed or composite tumours with an admixture of glandular and endocrine components that are present in separately identifiable components; collision tumours, wherein the 2 components are distinct and juxtaposed; and amphicrine cell tumours, which are predominantly composed of cells exhibiting dual endocrine and non-endocrine differentiation. Composite tumours are believed to arise from multipotent stem cells, via subsequent multidirectional differentiation after carcinogenesis. Hence, composite tumours are considered to contain a gradual transitional area between the adenocarcinoma and neuroendocrine component, and neuroendocrine markers, such as synaptophysin and chromogranin A, are found in some adenocarcinoma areas [6, 8]. In contrast, collision tumours are reportedly derived from 2 separate tumours located in close proximity, with no mixed or transitional areas [9]. Furthermore, amphicrine tumours are thought to represent another phenotype of composite tumours and are characterised by the appearance of dual neuroendocrine and glandular features in the same cell. This type of tumour is extremely rare, with only a few such reports in the stomach, pancreas, and liver $[4,10,11]$.

In the present case, the pathological findings of the liver metastatic lesion were consistent with those of the primary tumour. However, the cholangiocarcinoma-like tumour adjacent to the metastatic lesion exhibited gland formation, comprising atypical columnar epithelium, similar to that in a well-differentiated adenocarcinoma. Although the carcinoma cells, as well as the metastatic lesion, were positive for synaptophysin and chromogranin A on IHC staining, the expression of CK19 was diffusely positive in the cholangiocarcinomalike tumour, indicating a clear difference with the neuroendocrine component; moreover, this positive staining was not observed in the primary tumour. However, no gradual transitional area was observed between the adenocarcinoma and neuroendocrine component, and each component was not intermingled. According to Lewin's classification, the cholangiocarcinoma-like tumour has amphicrine properties, suggesting that the micrometastasis of pure neuroendocrine tumour may have acquired features of multipotent stem cells and differentiated into adenocarcinoma through the process of metastasis, or that the tumour with amphicrine properties newly arose from the liver as a primary tumour, such as in cases of collision tumours.

CK19 is normally expressed in ductal epithelium, including the bile duct, pancreas, and mucosa of the gastrointestinal tract. CK19 has primarily been used in diagnostic pathology to confirm the epithelial phenotype or clarify biliary and pancreatic ductular origin, usually in combination with various markers [12]. In the liver, CK19 may serve as a marker of biliary differentiation and hepatic progenitor cell features [13]. In general, intrahepatic cholangiocarcinoma is the most commonly occurring tumour with diffuse positivity for CK19 in primary liver tumours. Meanwhile, neuroendocrine tumours of the gastrointestinal tract are reported to be frequently positive for CK19, whereas interestingly, such tumours in the rectum are frequently negative for CK19 $[14,15]$. Based on these findings and the fact that the primary rectal tumour in the present case showed negative results for CK19, despite the diffusely positive staining in the cholangiocarcinoma-like tumour, we believe that the cholangiocarcinoma-like tumour in the present case may have represented a phenotype of intra- 
hepatic cholangiocarcinoma arising from the bile duct epithelium. The presence of a bile duct within this cholangiocarcinoma-like tumour, as well as the region of transition between it and the bile duct may support this finding. Thus, it is possible that the neuroendocrine tumour could potentially induce carcinogenesis to the neighbouring epithelium. Therefore, the phenotype of the exocrine component of the mixed tumour may depend on the neighbouring epithelium of the neuroendocrine tumour, such as in cases of squamous cell carcinoma in oesophageal or anal mixed tumours or adenocarcinoma in the mixed tumours of stomach, colon, and pancreas [4].

In summary, we present a case of a cholangiocarcinoma-like tumour exhibiting amphicrine properties in the liver metastatic site of a rectal neuroendocrine tumour. To our knowledge, no study has discussed a case of an adenocarcinoma component with amphicrine properties in a neuroendocrine tumour, which were observed only in the metastatic lesion and not in the primary lesion. Based on our findings, we suggest that the mechanism may involve the pathogenesis of a mixed tumour in the neuroendocrine tumour.

\section{Statement of Ethics}

Written informed consent was obtained from the patient.

\section{Disclosure Statement}

The authors have no conflicts of interest to declare.

\section{References}

1 Lawrence B, Gustafsson BI, Chan A, Svejda B, Kidd M, Modlin IM: The epidemiology of gastroenteropancreatic neuroendocrine tumors. Endocrinol Metab Clin North Am 2011;40:1-18, vii.

-2 Karakus E, Helvaci A, Ekinci O, Dursun A: Comparison of WHO 2000 and WHO 2010 classifications of gastroenteropancreatic neuroendocrine tumors. Turk J Gastroenterol 2014;25:81-87.

3 Karkouche R, Bachet JB, Sandrini J, Mitry E, Penna C, Cote JF, Blons H, Penault-Llorca F, Rougier P, Saint Andre JP, Emile JF: Colorectal neuroendocrine carcinomas and adenocarcinomas share oncogenic pathways. A clinico-pathologic study of 12 cases. Eur J Gastroenterol Hepatol 2012;24:1430-1437.

-4 La Rosa S, Marando A, Sessa F, Capella C: Mixed adenoneuroendocrine carcinomas (MANECs) of the gastrointestinal tract: an update. Cancers 2012;4:11-30.

-5 Vanacker L, Smeets D, Hoorens A, Teugels E, Algaba R, Dehou MF, De Becker A, Lambrechts D, De Greve J: Mixed adenoneuroendocrine carcinoma of the colon: molecular pathogenesis and treatment. Anticancer Res 2014;34:5517-5521.

-6 Bartley AN, Rashid A, Fournier KF, Abraham SC: Neuroendocrine and mucinous differentiation in signet ring cell carcinoma of the stomach: evidence for a common cell of origin in composite tumors. Hum Pathol 2011;42:1420-1429.

7 Lewin K: Carcinoid tumors and the mixed (composite) glandular-endocrine cell carcinomas. Am J Surg Pathol 1987;11(suppl 1):71-86.

-8 Fukui H, Takada M, Chiba T, Kashiwagi R, Sakane M, Tabata F, Kuroda Y, Ueda Y, Kawamata H, Imura J, Fujimori T: Concurrent occurrence of gastric adenocarcinoma and duodenal neuroendocrine cell carcinoma: a composite tumour or collision tumours? Gut 2001;48:853-856.

-9 Pecorella I, Memeo L, Ciardi A, Rotterdam H: An unusual case of colonic mixed adenoendocrine carcinoma: collision versus composite tumor. A case report and review of the literature. Ann Diagn Pathol 2007;11:285-290.

10 Jain D, Eslami-Varzaneh F, Takano AM, Ayer U, Umashankar R, Muller R, Klimstra DS: Composite glandular and endocrine tumors of the stomach with pancreatic acinar differentiation. Am J Surg Pathol 2005;29:1524-1529. 


\section{Case Reports in \\ Gastroenterology}

\begin{tabular}{l|l}
\hline Case Rep Gastroenterol 2016;10:431-439 \\
\hline $10.1159 / 000446641$ & $\begin{array}{l}\text { @ 2016 The Author(s). Published by S. Karger AG, Basel } \\
\text { www.karger.com/crg }\end{array}$ \\
\hline
\end{tabular}

Ishii et al.: Presence of Cytokeratin 19-Expressing Cholangiocarcinoma-Like Tumour in a Liver Metastatic Lesion of Rectal Neuroendocrine Tumour

11 Ikegami T, Kayashima H, Sadanaga N, Morizono S, Nakashima A, Matsuura H, Shirabe K, Maehara Y: Composite small cell and mucinous carcinoma originating from the intrahepatic bile duct: report of a case. Surg Today 2013;43:194-198.

12 Chu PG, Weiss LM: Keratin expression in human tissues and neoplasms. Histopathology 2002;40:403439.

13 Libbrecht L, Roskams T: Hepatic progenitor cells in human liver diseases. Semin Cell Dev Biol 2002;13:389-396.

14 Chu PG, Lau SK, Weiss LM: Keratin expression in endocrine organs and their neoplasms. Endocr Pathol 2009;20:1-10.

15 Chetty R, Serra S, Jain R, Evans M, Cooper K, Iyer A: CK19 immunoexpression in extrapancreatic endocrine tumours of the gastrointestinal tract. Histopathology 2009;55:116-120.

Ken Shirabe and Hiroyuki Kuwano contributed equally to this work
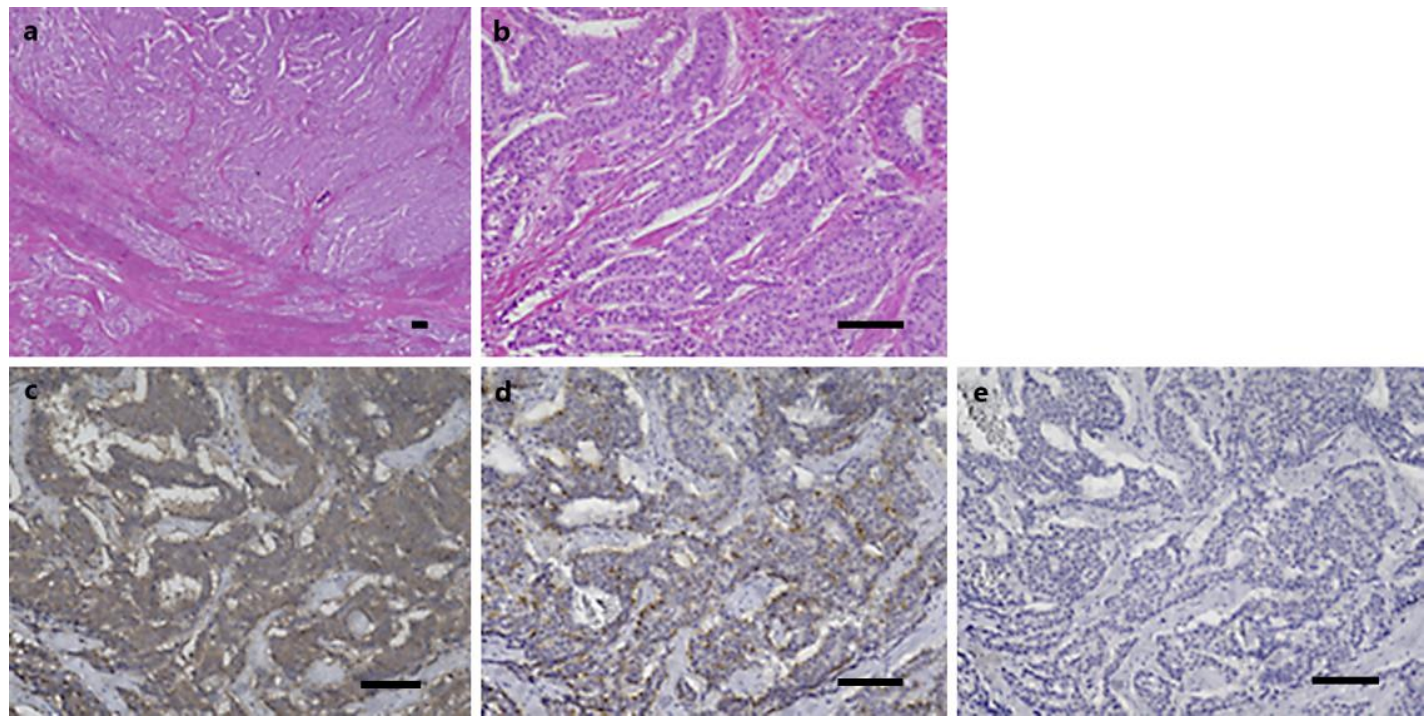

Fig. 1. Pathological findings of the primary rectal tumour. a, b Representative lesion of the primary tumour, and HE staining in low- and high-powered fields (original magnification, $\times 40$ and $\times 200$, respectively). c, d IHC staining of synaptophysin and chromogranin A; the rectal tumour shows positive results for synaptophysin (c) and chromogranin A (d) (original magnification, $\times 200$ ). e The Ki-67 labelling index is $<1 \%$ (original magnification, $\times 200$ ). Scale bars $=100 \mu \mathrm{m}$. 


\section{Case Reports in Gastroenterology} Rep Gastroenterol 2016;10:431-439 $10.1159 / 000446641$

Ishii et al: Presence of Cytokeratin 19-Expressing Cholangiocarcinoma-Like Tumour in a Liver Metastatic Lesion of Rectal Neuroendocrine Tumour

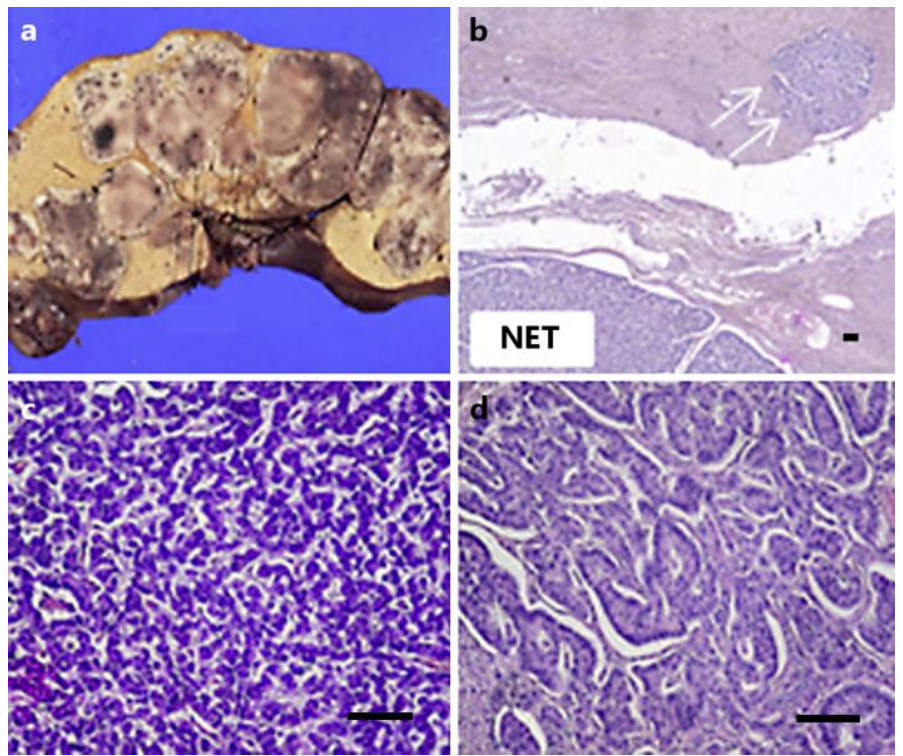

Fig. 2. Macro- and microscopic findings of liver metastatic lesions. The cholangiocarcinoma-like tumour is located adjacent to the metastatic lesion. a Gross appearance of the surgically resected liver specimen. Multiple nodules of various sizes are observed in the liver. $\mathbf{b}$ The positional relationship between the neuroendocrine tumour (NET) and microscopic cholangiocarcinoma-like tumour (arrows) (original magnification, $\times 40$ ). c, d HE staining of the liver metastatic lesion (c) and cholangiocarcinoma-like tumour (d). The cholangiocarcinoma-like tumour shows focal gland formation, such as in well-differentiated adenocarcinoma (original magnification, $\times 200$ ). Scale bars $=100 \mu \mathrm{m}$. 


\section{Case Reports in Gastroenterology}

a Liver metastatic lesion

synaptophysin

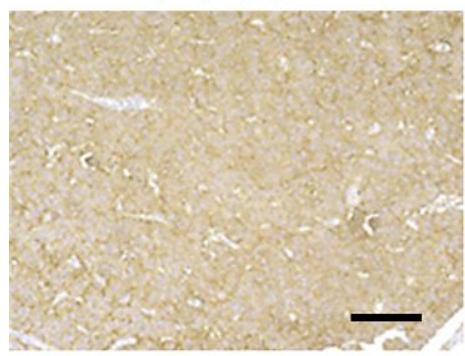

chromogranin A

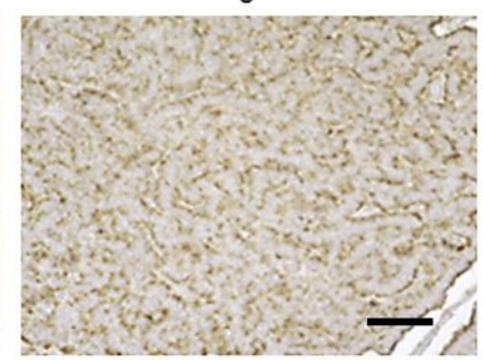

p53

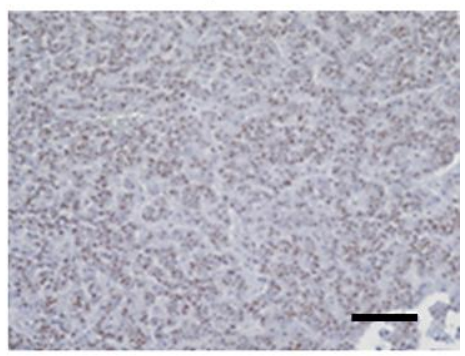

\section{b Cholangiocarcinoma-like tumor}

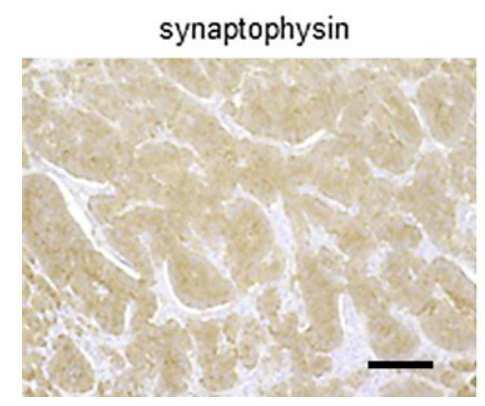

chromogranin A

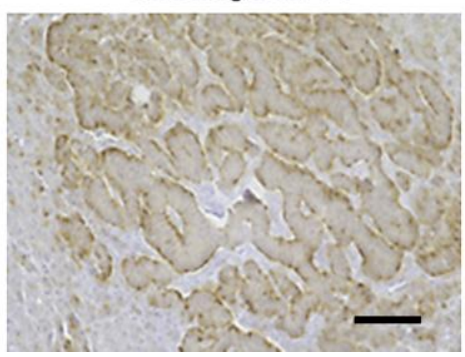

p53

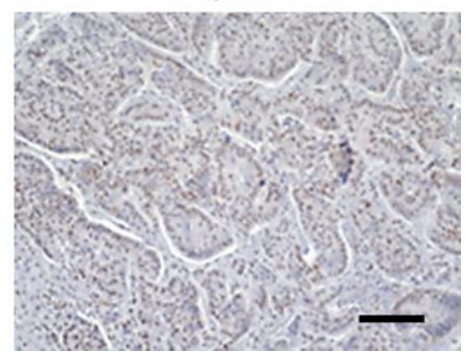

Fig. 3. Expression of neuroendocrine markers and p53 in the liver metastatic lesion (a) and cholangiocarcinoma-like tumour (b) on IHC staining. Both the liver metastatic lesion and cholangiocarcinoma-like tumour show positive results for synaptophysin (left panel), chromogranin A (middle panel), and p53 (right panel) (original magnification, $\times 200$ ). Scale bars $=100 \mu \mathrm{m}$. 


\section{Case Reports in \\ Gastroenterology}

Case Rep Gastroenterol 2016;10:431-439

(c) 2016 The Author(s). Published by S. Karger AG, Basel www.karger.com/crg

Ishii et al:: Presence of Cytokeratin 19-Expressing Cholangiocarcinoma-Like Tumour in a Liver Metastatic Lesion of Rectal Neuroendocrine Tumour
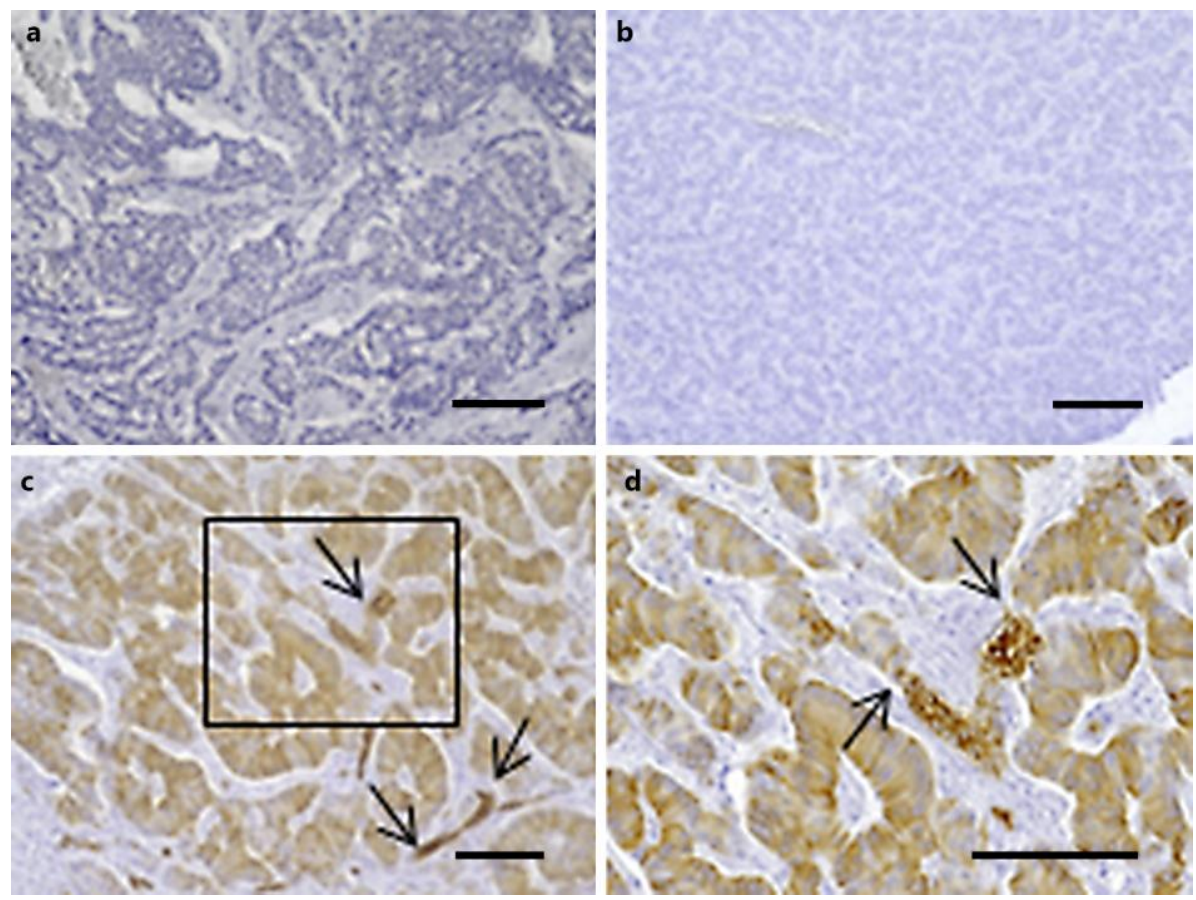

Fig. 4. Comparison of the expression of ductal marker cytokeratin 19 in the primary tumour, liver metastatic lesion, and cholangiocarcinoma-like tumour via immunohistochemical staining. a The primary tumour exhibits negative results for cytokeratin 19 staining. b The liver metastatic lesion also exhibits negative results for cytokeratin 19 staining. c The cholangiocarcinoma-like tumour is diffusely positive for cytokeratin 19. A normal bile duct that is positive for cytokeratin 19 is observed within the tumour (arrows). d High-powered field of $\mathbf{c}$ showing a region of transition between bile duct and cholangiocarcinoma-like tumour (arrows) (a-c original magnification, $\times 200$; $\mathbf{d}$ original magnification, $\times 400$ ). Scale bars $=100 \mu \mathrm{m}$. 